\title{
Post-traumatic ankle osteoarthritis: quality of life, frequency and associated factors
}

\author{
R. D’Ambrosi, C. Di Silvestri, L. Manzi, C. Indino, C. Maccario, F.G. Usuelli \\ IRCCS Istituto Ortopedico Galeazzi, Milan, Italy
}

\author{
CORRESPONDING AUTHOR: \\ Riccardo D'Ambrosi \\ IRCCS Istituto Ortopedico Galeazzi \\ Via Riccardo Galeazzi 4, \\ 20161 Milano MI \\ Phone: +39 3397066151 \\ E-mail: riccardo.dambrosi@hotmail.it
}

DOI:

10.32098/mltj.03.2019.10

LEVEL OF EVIDENCE: 4

\begin{abstract}
SUMMARY
Background. To assess the quality of life in patients with post-traumatic ankle osteoarthritis (PTOA) and evaluate frequency and relationship between dependent and independent factors. Methods. We performed a prospective study of patients with post-traumatic ankle osteoarthritis analyzing American Orthopaedic Foot and Ankle Society (AOFAS) ankle-hindfoot score, VAS, Short-Form Health Survey (SF-12) divided into mental (MCS) and physical (PCS) component.

Results. Our sample consisted of 258 patients, 162 (62.79\%) males and 96 (37.21\%) females,

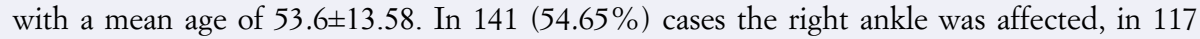
$(45.35 \%)$ the left. Overweight patients (BMI 25.0-29.99) was the predominant class $(43 \%)$. PCS and MCS resulted respectively 33.15 and 41.53 . We found a positive correlation between PCS and AOFAS ( $\mathrm{p}$-value $=0.02644 \mathrm{R}=0.14$ ) and a negative correlation between AOFAS and VAS ( $\mathrm{p}$-value $<0.0001, \mathrm{R}=-0.30$ ), VAS and PCS ( $\mathrm{p}$-value $=0.0109, \mathrm{R}=-0.16$ ) and MCS and PCS ( $\mathrm{p}$-value $=0.02696, \mathrm{R}=-0.14$ ).

Conclusions. PTOA impact patient's quality of life in an important way with limitations in daily activities. Around the age of 35, the physical and mental values of SF-12 tend to be very different. Almost half of the patients were overweight, while we did not find any differences as regards the affected side.
\end{abstract}

KEY WORDS

ankle osteoarthritis; body mass index; epidemiology; pain; quality of life

\section{INTRODUCTION}

Ankle osteoarthritis (OA) has a post-traumatic etiology in most cases, with pain and functional impairment that can develop from the initial trauma even years after; in particular the trauma occurs at the level of the talocrural joint (1-3). Unlike knee and hip, in which only in $5-10 \%$ of cases the etiology is post-traumatic, in the ankle this percentage rises to $90 \%(4-8)$. The ankle, in fact, is the joint most subjected to trauma during sports activities, with more than 300,000 injuries each year reported in the US, and an incidence of $52.3 / 1000$ sprains in athletes (9). This percentage is in fact an underestimation, indeed, an observational study of 10,393 basketball players reported that over half of ankle sprains were not reported or treated by medical personnel (10). Any traumatic event can compromise the articular surface of the ankle joint leading to post-traumatic OA. Alteration of ankle biomechanics, in turn, alters the mechanical loading of the ankle joint, which ultimately produces a mechanically driven degenerative remodeling process (11).
In patients undergoing total ankle replacement, more than $75 \%$ reported a history of at least one ankle sprain, with an economic burden associated with post-traumatic ankle osteoarthritis (PTOA) (12). In recent years national registries have shown a significant increase in the number of total ankle replacements implanted (13). Furthermore, patients with PTOA aree on average 14 years younger than patients with knee or hip osteoarthritis, and a rapid clinical deterioration, with pain, limitation of movement and functional impairment (14). Currently, there are no medical or surgical therapies to prevent or delay OA of the ankle and studies in this regard are limited, as shown by a recent meta-analysis, which highlights how the researchers' attention is more focused on other joints of the lower limb (15). The reason for these differences with the other joints is due to the micro-structure of the ankle joint, in fact the average thickness of the talar articular cartilage is approximately $0.89 \mathrm{~mm}$, significantly lower than other joints, with significant differences in the rate of extracellular matrix turnover and collagen composition (16). 
The aim of the study was to assess the quality of life (QOL) in patients with PTOA, and evaluate frequency and relationship between dependent and independent factors.

\section{METHODS}

After obtaining institutional review board approval, we performed a prospective study of the medical records of a number of consecutive patients presenting at our office with symptomatic ankle OA diagnosed clinically and radiographically by plain radiograph between September 1, 2013 and March 31, 2018. The inclusion criteria for this prospective case series were: patients with PTOA, with at least a history of trauma to the affected ankle. We considered as PTOA every form of OA developed to secondary joint trauma, with clinical signs of pain and dysfunction often lagging years or decades behind the initiating injury.

Exclusion criteria were: haemophilia, rheumatoid arthritis, severe metabolic disorders, autoimmune disease, ongoing chemotherapy, radiation treatment or immunosuppression; moreover we excluded patients subjected to intra-articular infiltrations in the last 6 months or patients who used painkillers within the previous 7 days.

All patients completed the following evaluations: American Orthopaedic Foot and Ankle Society (AOFAS) ankle-hindfoot score, Visual Analogue Pain Score (VAS), Short Form Health Survey (SF-12) (17-19). In addition the body height and weight of each patient were measured to calculate BMI. SF-12 is a generic questionnaire on quality of life that is not disease, age, or treatment specific (18). It evaluates the subjective perception of the individual in relation to the concepts of health and wellness. To simplify, the assessment is divided into a Physical Component Summary (PCS) and Mental Component summary (MCS). Moreover, we compared the results obtained from our cohort, the SF-12, with a sample of the Italian population to assess the differences found (18). The AOFAS is one of the most widely used clinician-reporting tools for foot and ankle conditions, developed in 1994 (17). The Survey include subjective and objective questions regarding pain, deformity and function. VAS consists of a straight line with the endpoints defining extreme limits such as 'no pain at all' and 'pain as bad as it could be'. The patient is asked to mark their pain level on the line between the two endpoints. The distance between 'no pain at all' and the mark then defines the subject's pain (19).

Moreover, sex, age, BMI and affected size for each patient were recorded and in order to evaluate incidence, frequency and correlations.

This prospective study was conducted on the basis of the STROBE statement guidelines (20). Informed consent was obtained from all participants, and procedures were conducted according to the Declaration of Helsinki. This research protocol has been approved by the local ethics committee (21).

\section{Statistical Analysis}

The statistical analysis was performed by SPSS and Excel using PIVOT tables. We evaluated significant correlation with Pearson's linear correlation coefficient R. Specifically, to determine if the correlation was significant, the test $t$ was computed by comparing the obtained $t$ value with the critical value of $\mathrm{n}-2$ degrees of freedom, taking into account the bi-directional hypothesis considering as significant a $\mathrm{p}$-value $<0.05$. We also studied the associations of variables by comparing the chi-square value with those tabulated, allowing us to assume that the difference between the two groups is significant at the $5 \%$ probability level. Instead of performing the variance analysis, we used the "t-test". In this case, the p-value (calculated with respect to the random variable $t$ ) and the averages of the various groups is reported. Descriptive analysis was performed to identify the overall PTOA frequency in the study sample, and the frequency was stratified by age, gender and clinical parameters.

\section{Results}

Of 290 patients screened for eligibility, 258 satisfied the inclusion criteria and were enrolled in the study. Of the 32 excluded patients, 9 had infiltrated over the year before clinical evaluation, 10 had rheumatoid arthritis, 5 were haemophilic, 6 women were pregnant and 2 were in chemotherapy treatment. In our study, our sample consists of 258 patients, consisting of $162(62.79 \%)$ males and $96(37.21 \%)$ females, with a range of 21 to 87 years (average age 53.5) for males, range 24 to 81 years (average age 53.4) for females. In $141(54.65 \%)$ cases the right ankle was affected, in 117 $(45.35 \%)$ the left.

\section{Frequency}

To better understand the frequency of PTOA we divided the AOFAS, VAS, age and BMI variables into subgroups as follows:
Age:
1) Young [21-39]
2) Middle Age [40-59]
3) Elderly [60-87]
VAS:
1) Low pain [0-3]
2) Medium pain [4-7]
3) High pain [8-10] 


\section{BMI:}

1) Underweight $[<18,50]$

2) Normal weight $[18,50-24,99]$

3) Overweight $[25,0$-29, 99]

4) Obese Class I [30, $0-34,99]$

5) Obese Class II $[35,0-39,99]$

6) Obese Class III $[\geq 40,0]$

\section{AOFAS:}

1) Low [0-19]

2) Medium [20-39]

3) High [40-59]

4) Very High $[\geq 60]$

We found a higher incidence for male than female, while the ratio between right and left side was similar. It is interesting to see, as the predominant class is overweight, representing almost $43 \%$ of the patients who summed up the first two classes, make up $75 \%$ of the total cohort of patients, while only $25.6 \%$ of the patients belong to the obese class (I, II or III). Moreover as far as age is concerned, most patients $(81 \%)$ are over 40 years of age. Results are summarized in Table I.

\section{Gender, age and VAS}

Table II shows how many males and females are present for every class of age, revealing a prevalence in the male group aged $40-59$ years old $(27.13 \%$ in the total group, and $43.20 \%$ in the male group). Only $19.0 \%$, of both sexes have ankle problems with an age below 40 . There is no difference between the gender in the different age categories $(\mathrm{p}<0.05)$. Of the 162 male individuals, $96.91 \%$ have medium or high pain of the ankle, of which $65.43 \%$ refers to severe ankle pain. In the female group there was no one with low pain, and the relationship of women with strong pain is similar to that of men $(70.83 \%)$. These values are similar for both gender in their VAS classes $(\mathrm{p}<0.05)$. Results are reported in Table II.

\section{BMI, gender and age}

We found many patients belonging to the middle class of age (40-59 y.o.) especially in overweight patients (53 - 18.92\% marked with * in table III). As the degree of obesity increases, the number of individuals decreases, although this is significant because $25.58 \%$ of our patients have a BMI

Table I. Clinical and demographic parameters score for patient described by mean, standard deviation and distribution

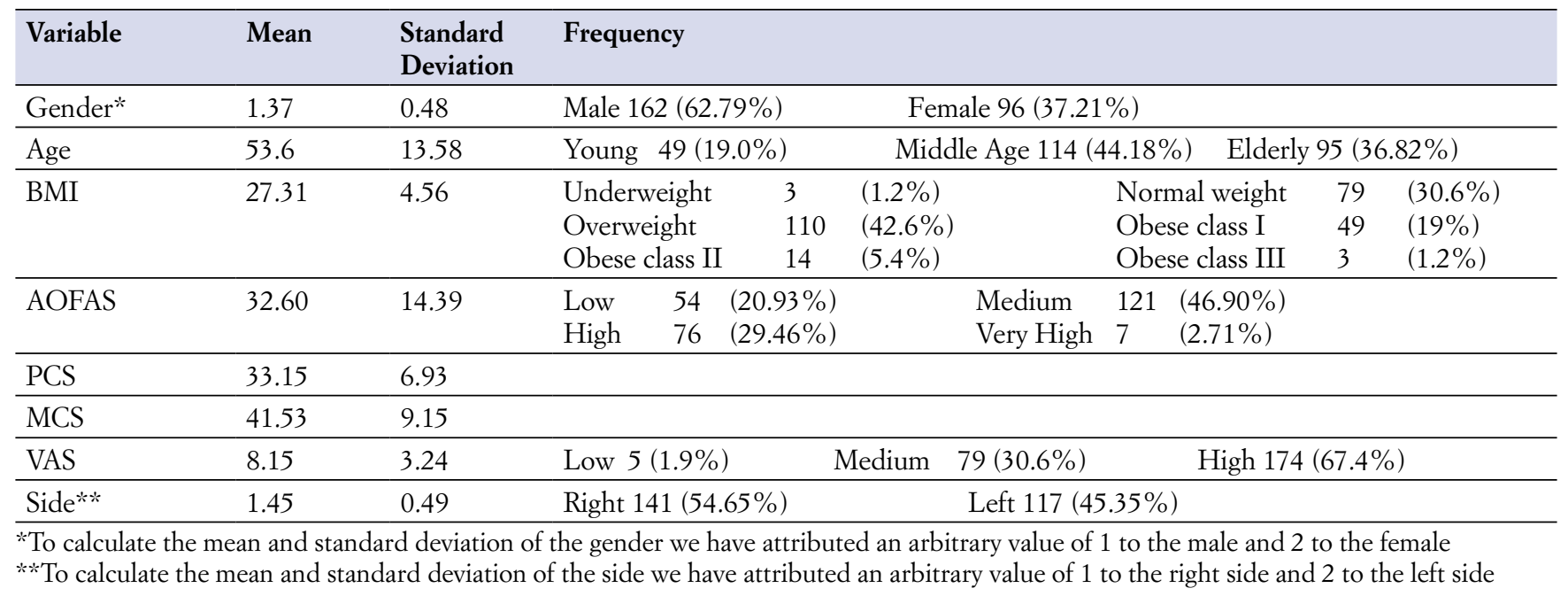

Table II. Frequency in the different categories of VAS and age divided by gender

\begin{tabular}{lllllllll}
\hline Gender & \multicolumn{3}{c}{ VAS } & \multicolumn{3}{c}{ Age } \\
\hline & Low & Medium & High & Total & $21-39$ & $40-59$ & $60-87$ & Total \\
\hline Male & 5 & 51 & 106 & 162 & 31 & 70 & 61 & 162 \\
\hline Female & 0 & 28 & 68 & 96 & 18 & 44 & 34 & 96 \\
\hline Total & 5 & 79 & 164 & 258 & 49 & 114 & 95 & 258 \\
\hline
\end{tabular}


level with at least Obesity level Class I (66). Considering gender we noted that there is no difference in normal weight patients between 40 and 59 years ( 15 males - 15 females); the only class in which the number of women is greater than men is in the older group with a level of obesity II ( 2 males - 4 females). Results are reported in table III.

\section{AOFAS, side and BMI frequency}

Almost half of the patients $(121-46.90 \%)$ report a medium value of AOFAS. There is no difference between the pain side $(54.65 \%$ right side and $45.35 \%$ left side). Despite the not significant difference in "side" we almost never find a strong prevalence of the left side except in some cases (marked with *). Only $1 \%$ of those who have ankle pain are in the underweight category. Results are summarized in table IV.

Table III. Absolute frequency between BMI and age

\begin{tabular}{|c|c|c|c|c|}
\hline & AGE & & & \\
\hline BMI & $\begin{array}{l}\text { Young age } \\
{[21,39]}\end{array}$ & $\begin{array}{l}\text { Middle age } \\
{[40,59]}\end{array}$ & $\begin{array}{l}\text { Elder } \\
{[60,87]}\end{array}$ & Total \\
\hline \multicolumn{5}{|l|}{ MALE } \\
\hline UW & 1 & 1 & 1 & 2 \\
\hline NW & 13 & 15 & 18 & 46 \\
\hline OW & 11 & $34 *$ & 29 & 74 \\
\hline I & 4 & 16 & 10 & 30 \\
\hline II & 2 & 4 & 2 & 8 \\
\hline III & 1 & I & 1 & 2 \\
\hline \multirow[t]{2}{*}{ Sub Total } & 31 & 70 & 61 & 162 \\
\hline & {$[21,39]$} & {$[40,59]$} & {$[60,87]$} & Total \\
\hline \multicolumn{5}{|l|}{ FEMALE } \\
\hline \multicolumn{5}{|l|}{ BMI } \\
\hline UW & 1 & I & I & 1 \\
\hline NW & 5 & 15 & 13 & 33 \\
\hline OW & 6 & $19 *$ & 11 & 36 \\
\hline $\mathrm{I}$ & 4 & 9 & 6 & 19 \\
\hline II & 1 & 1 & 4 & 6 \\
\hline III & 1 & I & I & 1 \\
\hline Sub Total & 18 & 44 & 34 & 96 \\
\hline Total & 49 & 114 & 95 & 258 \\
\hline
\end{tabular}

UW=underweight; NW=normal weight; $\mathrm{OW}=$ overweight; $\mathrm{I}=$ obese class I; II=obese class II; III= obese class III

$*$ =higher values of our cohort of patients

\section{AOFAS, BMI and age}

Analyzing the AOFAS subgroups, by comparing young and middle age groups, we noted that the proportion initially is more than double (10:24 and 27:54), until almost triple (12:33). Specifically, among individuals aged between 21 and 39 years, there are 10 who scored AOFAS $=<19$. More than double (24) are present in the age class [40, 59]. Furthermore we found that the number of normal weight patients is always superior to obese class I patients except in patients 40-59 y.o. with high AOFAS: in fact in this case the situation is muted (9 individuals out of 33 are of obesity level I, marked with * in table $\mathrm{V}$, versus 7 individuals with a BMI score 18.50 - 24.99). Results are reported in Table $\mathbf{V}$.

Table IV. Absolute frequency between AOFAS, BMI and side

\begin{tabular}{|c|c|c|c|c|c|c|c|}
\hline & BMI & & & & & & \\
\hline AOFAS & UW & NW & OW & I & II & III & Totale \\
\hline \multicolumn{8}{|l|}{$\begin{array}{l}\text { Low } \\
(0-19) \\
\end{array}$} \\
\hline $\mathrm{R}$ & I & 10 & 13 & 6 & 2 & I & 31 \\
\hline $\mathrm{L}$ & $1 *$ & 7 & 9 & 3 & 2 & 1 & 23 \\
\hline 1 Total & 1 & 17 & 22 & 9 & 4 & 1 & 54 \\
\hline \multicolumn{8}{|l|}{$\begin{array}{l}\text { Medium } \\
(20-39) \\
\end{array}$} \\
\hline $\mathrm{R}$ & 1 & 27 & 27 & 10 & 2 & & 67 \\
\hline $\mathrm{L}$ & / & 11 & 26 & 10 & $5 *$ & 2 & 54 \\
\hline 2 Total & 1 & 38 & 53 & 20 & 7 & 2 & 121 \\
\hline
\end{tabular}

High

(40-59)

\begin{tabular}{lllllll}
\hline $\mathrm{R}$ & $/$ & 13 & 16 & 9 & 1 & 39 \\
\hline $\mathrm{L}$ & $1^{*}$ & 10 & 16 & 8 & 2 & 37 \\
\hline 3 Total & $\mathbf{1}$ & $\mathbf{2 3}$ & $\mathbf{3 2}$ & $\mathbf{1 7}$ & $\mathbf{3}$ & $\mathbf{7 6}$ \\
\hline
\end{tabular}

Very High

$(\geq 60)$

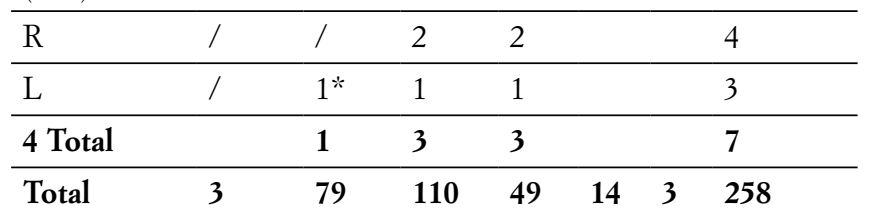

$\mathrm{UW}=$ underweight; $\mathrm{NW}=$ normal weight; $\mathrm{OW}=$ overweight; $\mathrm{I}=$ obese class I; II=obese class II; III= obese class III

$*=$ Values in which the incidence of the left side is greater than the right 
Table V. Absolute frequency between AOFAS, BMI and age

\begin{tabular}{|c|c|c|c|c|c|c|c|}
\hline AOFAS & UW & NW & OW & $\mathbf{I}$ & II & III & Totale \\
\hline \multicolumn{8}{|l|}{ Low AOFAS [0-19] } \\
\hline Young age $[21,39]$ & 1 & 4 & 2 & 2 & 1 & 1 & 10 \\
\hline Middle age $[40,59]$ & 1 & 8 & 11 & 3 & 1 & 1 & 24 \\
\hline Elder $[60,87]$ & 1 & 5 & 9 & 4 & 2 & 1 & 20 \\
\hline Low - Total & 1 & 17 & 22 & 9 & 4 & 1 & 54 \\
\hline \multicolumn{8}{|c|}{ Medium AOFAS [20-39] } \\
\hline Young age $[21,39]$ & 1 & 10 & 10 & 3 & 2 & 1 & 27 \\
\hline Middle age $[40,59]$ & 1 & 15 & 26 & 11 & 2 & 1 & 54 \\
\hline Elder $[60,87]$ & 1 & 13 & 17 & 6 & 3 & 1 & 40 \\
\hline Medium- Total & 1 & 38 & 53 & 20 & 7 & 2 & 121 \\
\hline \multicolumn{8}{|c|}{ High AOFAS [40-59] } \\
\hline Young age $[21,39]$ & 1 & 4 & 5 & 3 & 1 & 1 & 12 \\
\hline Middle age $[40,59]$ & 1 & 7 & 15 & $9 *$ & 2 & 1 & 33 \\
\hline Elder $[60,87]$ & 1 & 12 & 12 & 5 & 1 & 1 & 31 \\
\hline High - Total & 1 & 23 & 32 & 17 & 3 & 1 & 76 \\
\hline \multicolumn{8}{|c|}{ Very High AOFAS $[\geq 60]$} \\
\hline Middle Age $[40,59]$ & 1 & 1 & 1 & 2 & 1 & 1 & 3 \\
\hline Elder $[60,87]$ & 1 & 1 & 2 & 1 & 1 & 1 & 4 \\
\hline Very High - Total & 1 & 1 & 3 & 3 & 1 & 1 & 7 \\
\hline Total & 3 & 79 & 110 & 49 & 14 & 3 & 258 \\
\hline
\end{tabular}

UW=underweight; NW=normal weight; OW=overweight; I=obese class I; II=obese class II; III= obese class III

*=Values in which the incidence of overweight or obese patietns is greater than normal weight patients

\section{Subjective health status assessment}

Considering the physical and the component score of the SF-12 we observed that the range respectively resulted:

PCS $\rightarrow$ 19.4 -53.6

MCS $\rightarrow 17.6-71.4$

As regards PCS more than half of patients (138/258 $53.4 \%$ ) reported a value of less than 32.9. Of the remaining patients the majority focuses between a value of 33 and 40.3 . In PCS score, differently from MCS, many patients reported very low scores (figure 1).

In MCS about $60 \%$ of patients (163/258) are concentrated among the values of 35.1 and 46.6. The remaining patients are divided between lower values $(15.11 \%)$ and higher $(21.7 \%)$ in a fairly homogeneous way (figure 2$)$.

\section{Comparison between MCS and PCS by age}

We noted that PCS-MCS values initially are closer to each other; among individuals over the age of 35 the difference between the two scores increases. Mostly, patients who have

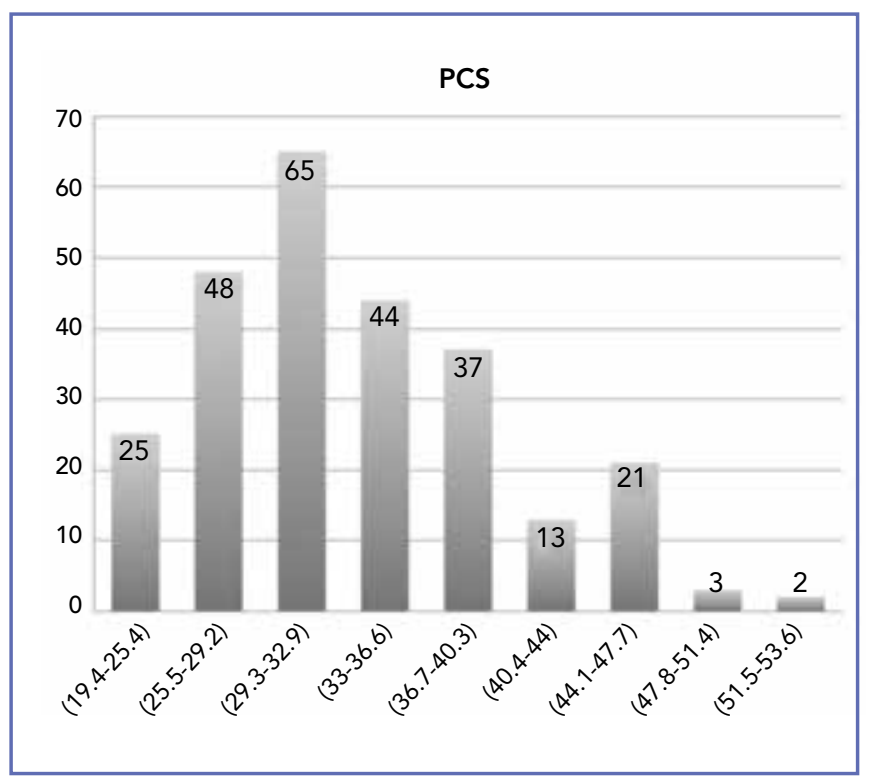

Figure 1. The chart shows the distribution of PCS values in our sample 


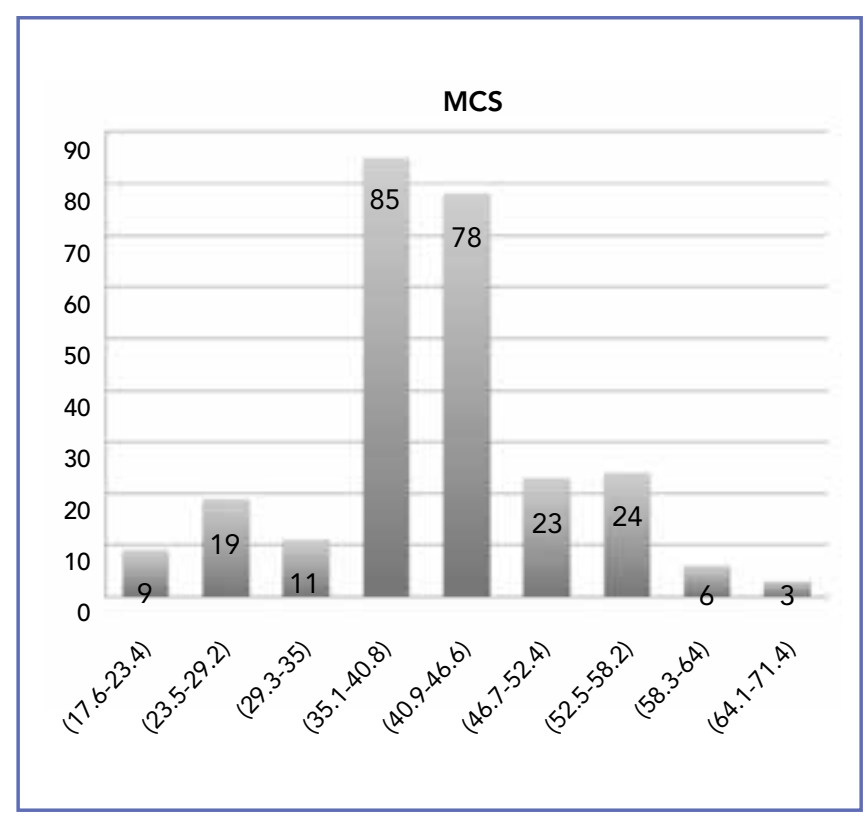

Figure 2. The chart shows the distribution of MCS values in our sample

PTOA report higher mental score than physical. As the age increases, the difference between the two indexes increases and then mean values return similar to the initial situation.

\section{Comparison Between groups}

\section{Age}

The initial sample of 258 patients was divided into two groups, defining the group of patients under 50 , (i.e., those of less than or equal to 50 years), consisting of 110 patients, $38 \%$ females and $62 \%$ males, with a mean age of 40.53 years $(\mathrm{SD} \pm 6.99)$ and the group of patients over 50 consist- ing of 148 patients, 36\% females and 64\% males, with a mean age of 63.09 years $(\mathrm{SD} \pm 8.19)$. The only statistically significant difference found was the MCS, higher in older patients $(\mathrm{p}<0.05)$.

\section{Gender}

We compared the clinical outcomes of the male group, with the female group and we found no significant difference between the two groups $(\mathrm{p}>0.05)$

\section{Side}

We compared the clinical outcomes of the patients with the right ankle affected, with the patients with left ankle affected and we found no significant difference between the two groups $(\mathrm{p}>0.05)$

\section{BMI}

We compared the clinical outcomes of patients with a BMI $<25$, with the patients with $\mathrm{BMI} \geq 25$ and we found no significant difference between the two groups $(p>0.05)$

\section{Correlations}

Analyzing the correlation between the variables we found an important positive correlation between PCS and AOFAS ( $\mathrm{p}$-value $=0.02644 \mathrm{R}=0.14)$ and a negative correlation between AOFAS and VAS ( $\mathrm{p}$-value $<0.0001, \mathrm{R}=-0.30$ ), VAS and PCS ( $\mathrm{p}$-value $=0.0109, \mathrm{R}=-0.16)$ and MCS and PCS ( $\mathrm{p}$-value $=0.02696, \mathrm{R}=-0.14)$. Correlations are reported in table VI.

\section{DISCUSSION}

The aim of the study was to assess the quality of life in patients with PTOA and evaluate the frequency of the pathology. In our cohort we found that PTOA affects male and female in a similar way, but we noted a high incidence in overweight

Table VI. Correlation between variables.

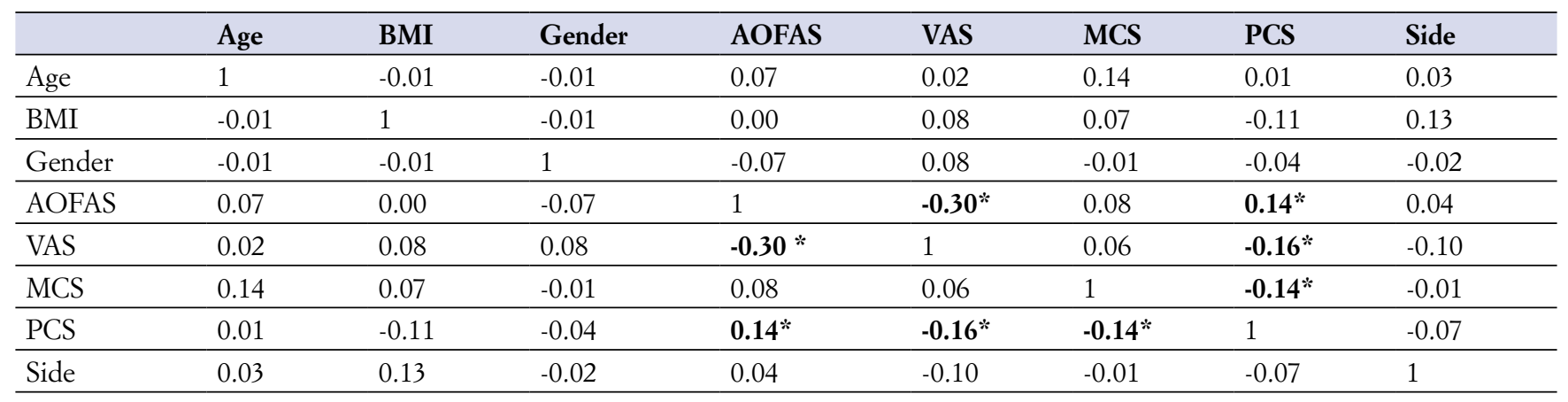

*significant correlation 
patients (BMI 25.0 - 29.99) and the relatively young (age 40 - 59). Even with regard to the concerned side we did not find any particular differences. As reported in a previous study, and confirmed by our results, individuals with ankle arthrosis tend to be younger than other patients with joint degeneration in the lower limbs and present faster functional loss, with progression to the final stages of the disease between 10 and 20 years after the start of the lesion. The post-traumatic etiology can be explained by the unique anatomic, biomechanical, and cartilage characteristics of the ankle $(1,14,15)$. In fact, the ankle presents a smaller contact area than the hip or knee, leading to a higher force per square centimetre $(22,23)$. Biomechanically, the ankle, with its high congruency, acts as a rolling joint with high congruency (22). The knee, however, acts as a rolling, sliding, and rotating joint during motion (24). This is the principal difference for increased cartilage degeneration and the high rate of primary OA in the knee. Moreover the cartilage of the ankle is thinner with respect to knee or hip, but owns a higher compressive stiffness, proteoglycan density, lower matrix degradation, and less response to catabolic stimulations (25-27).

The high incidence in young subjects with high functional demands, makes their quality of life much worse, in fact, in our sample we found the average values of MCS and PCS respectively of 41.54 and 33.15 and in particular we have seen how the physical component tends to split in respect to the mental component at around 35 years.

These results are supported by previous studies on the quality of life assessment in ankle osteoarthritis, in fact in 2008, Glazebrook evaluated the quality of life in 130 patients with end-stage ankle arthrosis with the Short Form-36 (SF-36). This cohort was compared with a similar cohort of 130 patients with end-stage hip arthrosis, and it was noted that patients with ankle arthrosis had significantly worse mental component summary scores, role-physical scores, and general health scores $(\mathrm{p}<0.05)(28)$.

Previously, Saltzman aimed to determine how morbidity unrelated to the ankle influences the perception of physical function and pain by patients with ankle osteoarthritis on 195 patients with ankle osteoarthritis (29). The author found that the perception of ankle pain by patients with ankle osteoarthritis is significantly and linearly associated with the number of other musculoskeletal problems (not related to the foot or ankle). Moreover the degree of physical impairment associated with ankle osteoarthritis, as measured with the SF-36, is equivalent to that reported to be associated with severely disabling medical problems including end-stage kidney disease and congestive heart failure (29).

Recently, Desai compared preoperative health-related quality of life (HRQoL) between patients with bilateral and those with unilateral end-stage ankle arthritis and demonstrated a low quality of life for both conditions, in particular a significantly greater morbidity in patients with end-stage bilateral ankle arthritis awaiting surgical intervention compared with patients with end stage unilateral arthritis awaiting surgical intervention (31).

Comparing our results with a national survey brought to light how severely PTOA impact quality of life. Specifically, in an Italian study of 10,546 persons with a mean age of 45-54 years, patients had a mean MCS of 49.85 and PCS of 50.94 (18).

In addition, comparing our SF-12 outcomes with some of the most common chronic diseases, the results are even more interesting: in fact, patients with diabetes report value of 45.11 and 40.22, respectively, for MCS and PCS, while as regards tumor mean results, respectively, were 41.64 and 37.87. The most significant values that we found regarded the physical component; in this case, the reference value that is closest to our group is in patients affected by tumors, which show a mean value of PCS of 37.87 or angina pectoris (36.17). Our results show that PTOA should be considered a serious disease with major social implications, such as cancer, heart disease or diabetes (18).

Another important result of our study is BMI, in fact more than $40 \%$ of patients are overweight, although we did not find any correlation between the BMI and the other factors studied.

Literature identifies BMI as one of the risk factors in the development of PTOA (31); in this respect it is very interesting to consider Coster's study. The author evaluated whether patients with ankle OA have higher bone mineral density, smaller bone size, and higher BMI than the general population and, if so, this would depend on differences in lifestyle or anthropometry (32). The study concluded that patients with $\mathrm{OA}$ in the ankle have higher bone mineral density and smaller bone size than that expected by their BMI. This phenotype may provide unfavourable forces across the joint and is hypothetically important for development of OA.

Moreover, in a recent study we have shown that as weight increases, there is an increase in osteochondral central talus lesions, confirming that weight is a negative risk factor in this regard (33).

Ankle arthritis is a major disease with a deep negative impact on a generally young and active population. Historically it has been treated with ankle fusion, which is a sort of sacrifice-solution, not very well-accepted by these patients.

More recently ankle replacement has been proposed as a reliable standard option (34-35).

In order to avoid ankle osteoarthritis, a number of studies have looked at realignment osteotomies as a possible temporary-limited solution, confirming the role of deformities on arthritis onset. 
We advocate further studies focusing on social costs of this pathology, which may provide an additional reason for strong research activity on both sides: replacement and osteotomies. This study presents some limitations. First of all we did not perform a sub-analysis considering the type of trau$\mathrm{ma}$; another limitation of the study is the lack of correlation between the type of deformity and associated factors. Furthermore, the lack of records concerning pre-injury diseases, since the evaluation of quality of life in this paper was focused on ankle disability due to post-traumatic etiol-

\section{REFERENCES}

1. Delco ML, Kennedy JG, Bonassar LJ, Fortier LA. Post-traumatic osteoarthritis of the ankle: A distinct clinical entity requiring new research approaches. J Orthop Res 2017; 35:440-53.

2. Carbone A, Rodeo S. Review of current understanding of post traumatic osteoarthritis resulting from sports injuries. J Orthop Res 2017; 35:397-405.

3. Bloch B, Srinivasan S, Mangwani J. Current Concepts in the Management of Ankle Osteoarthritis: A Systematic Review. J Foot Ankle Surg 2015; 54:932-939.

4. Brown TD, Johnston RC, Saltzman CL, Marsh JL, Buckwalter JA. Posttraumatic osteoarthritis: a first estimate of incidence, prevalence, and burden of disease. J Orthop Trauma. 2006;20:739-744.

5. Richmond SA, Fukuchi RK, Ezzat A, Schneider K, Schneider G, Emery CA. Are joint injury, sport activity, physical activity, obesity, or occupational activities predictors for osteoarthritis? A systematic review. J Orthop Sports Phys Ther. 2013;43:515-B519.

6. Weatherall JM, Mroczek K, McLaurin T, et al. Post-traumatic ankle arthritis. Bull Hosp Jt Dis. 2013;71:104-112.

7. Wilson MG, Michet CJJ, Ilstrup DM, et al. Idiopathic symptomatic osteoarthritis of the hip and knee: a population-based incidence study. Mayo Clin Proc. 1990;65:1214-1221.

8. Valderrabano V, HorisbergerM, Russell I, Dougall H, Hintermann B. Etiology of ankle osteoarthritis. Clin Orthop Relat Res. 2008;467:1800-1806.

9. Mauntel TC, Wikstrom EA, Roos KG, Djoko A, Dompier TP, Kerr ZY.The Epidemiology of High Ankle Sprains in National Collegiate Athletic Association Sports. Am J Sports Med. 2017;45:2156-2163

10. McKay GD, Goldie PA, Payne WR, Oakes BW, Watson LF. A prospective study of injuries in basketbala total profile and comparison by gender and standard of competition. J Sci Med Sport. 2001; 4:196-211.

11. Ewalefo SO, Dombrowski M, Hirase T, Rocha JL, Weaver M, Kline A, Carney D, Hogan MV. Management of Posttraumatic Ankle Arthritis: Literature Review. Curr Rev Musculoskelet Med. 2018 Oct 16. doi: 10.1007/s12178-018-9525-9. [Epub ahead of print]

12. Gribble PA, Bleakley CM, Caulfield BM, Docherty CL, Fourchet F, Fong DT, et al. Evidence review for the 2016 International Ankle Consortium consensus statement on the prevalence, impact and long-term consequences of lateral ankle sprains. Br J Sports Med 2016; 50:1496-1505. ogy. Moreover only one clinical assessment did not allow us to measure over time the change of clinical parameters.

\section{CONCLUSIONS}

PTOA impact patients' quality of life, in an important way with limitations on daily activities. Around the age of 35, the physical and mental values of SF-12 tend to be very different. Almost half of the patients are overweight, while we did not find any differences as regards the affected side.

13. D’Ambrosi R, Banfi G, Usuelli FG. Total ankle arthroplasty and national registers: What is the impact on scientific production?_Foot Ankle Surg. 2018 Mar 6. pii: S1268-7731(18)300468. doi: 10.1016/j.fas.2018.02.016.

14. Barg A, Pagenstert GI, Hügle T, Gloyer M, Wiewiorski M, Henninger $\mathrm{HB}$, et al. Ankle osteoarthritis: etiology, diagnostics, and classification. Foot Ankle Clin. 2013; 18:411-426.

15. Lefèvre-Colau MM, Nguyen C, Haddad R, Delamarche P, Paris G, Palazzo C, Poiraudeau S, Rannou F, Roren A. Is physical activity, practised as recommended for health benefit, a risk factor for osteoarthritis? Ann Phys Rehabil Med. 2016;59:196-206.

16. Kraeutler MJ, Kaenkumchorn T, Pascual-Garrido C, Wimmer MA, Chubinskaya S. Peculiarities in Ankle Cartilage. Cartilage. 2017;8:12-18.

17. Leigheb M, Janicka P, Andorno S, Marcuzzi A, Magnani C, Grassi F. Italian translation, cultural adaptation and validation of the "American Orthopaedic Foot and Ankle Society's (AOFAS) ankle-hindfoot scale”. Acta Biomed. 2016;87:38-45.

18. Kodraliu G, Mosconi P, Groth N, et al. Subjective health status assessment: evaluation of the Italian version of the SF-12 Health Survey. Results from the MiOS Project. J Epidemiol Biostat. 2001;6:305-316

19. Wewers ME, Lowe NK. A critical review of visual analogue scales in the measurement of clinical phenomena. Res Nurs Health. 1990;13:227-236.

20. von Elm E, Altman DG, Egger M, Pocock SJ, Gøtzsche PC, Vandenbroucke JP; STROBE Initiative. The Strengthening the Reporting of Observational Studies in Epidemiology (STROBE) statement: guidelines for reporting observational studies. J Clin Epidemiol 2008; 61:344-9.

21. Padulo J, Oliva F, Frizziero A, Maffulli N. Muscles, Ligaments and Tendons Journal Basic principles and recommendations in clinicaland field Science Research: 2016 Update. Muscles Ligaments Tendons J. 2016;6:1-5.

22. Brockett CL, Chapman GJ. Biomechanics of the ankle. Orthop Trauma. 2016;30:232-238.

23. van Dijk CN, Reilingh ML, Zengerink M, van Bergen CJ. Osteochondral defects in the ankle: why painful? Knee Surg Sports Traumatol Arthrosc. 2010;18:570-580

24. Amano K, Li Q, Ma CB. Functional knee assessment with advanced imaging. Curr Rev Musculoskelet Med. 2016;9:123. 129.

25. Körner D, Gueorguiev B, Niemeyer P, Bangert Y, Zinser W, Aurich $\mathrm{M}$, et al. Parameters influencing complaints and joint 
function in patients with osteochondral lesions of the ankle-an investigation based on data from the German Cartilage Registry (KnorpelRegister DGOU). Arch Orthop Trauma Surg 2017;137: 367-373.

26. Schreiner MM, Mlynarik V, Zbýň Š, Szomolanyi P, Apprich S, Windhager R, et al. New Technology in Imaging Cartilage of the Ankle. Cartilage. 2017;8:31-41.

27. Gianakos AL, Yasui Y, Hannon CP, Kennedy JG. Current management of talar osteochondral lesions. World J Orthop. 2017;8:12-20.

28. Glazebrook M, Daniels T, Younger A, Foote CJ, Penner M, Wing K, Lau J, et al. Comparison of health-related quality of life between patients with end-stage ankle and hip arthrosis. J Bone Joint Surg Am. 2008; 90:499-505

29. Saltzman CL, Zimmerman MB, O'Rourke M, Brown TD, Buckwalter JA, Johnston R. Impact of comorbidities on the measurement of health in patients with ankle osteoarthritis. J Bone Joint Surg Am. 2006;88:2366-2372.

30. Desai SJ, Glazebrook M, Penner MJ, Wing KJ, Younger AS, Pinsker E, et al Quality of Life in Bilateral Vs. Unilateral End-Stage Ankle Arthritis and Outcomes of Bilateral Vs.
Unilateral Total Ankle Replacement. J Bone Joint Surg Am. 2017; 99:133-140.

31. Lübbeke A, Salvo D, Stern R, Hoffmeyer P, Holzer N, Assal M. Risk factors for post-traumatic osteoarthritis of the ankle: an eighteen year follow-up study. Int Orthop. 2012;36:1403-1410.

32. Cöster MC, Rosengren BE, Karlsson C, von Schevelow T, Magnusson H, Brudin L, et al. Bone mass and anthropometry in patients with osteoarthritis of the foot and ankle. Foot Ankle Surg. 2014;20:52-56.

33. D’Ambrosi R, Maccario C, Serra N, Ursino C, Usuelli FG. Relationship between symptomatic osteochondral lesions of the talus and quality of life, body mass index, age, size and anatomic location. Foot Ankle Surg. 2018;24:365-372.

34. Usuelli FG, Maccario C, D’Ambrosi R, Surace MF, Vulcano E. Age-Related Outcome of Mobile-Bearing Total Ankle Replacement._Orthopedics. 2017;40:e567-e573.

35. Total ankle replacement: is pre-operative varus deformity a predictor of poor survival rate and clinical and radiological outcomes?_Usuelli FG, Di Silvestri CA, D’Ambrosi R, Orenti A, Randelli F. Int Orthop. 2018 Oct 15. doi: 10.1007/s00264018-4189-z. 